\section{LETTERS \\ Regional cerebral glucose metabolism in akinetic catatonia and after remission}

K L Kahlbaum published in 1874 the first recorded description of catatonia. Akinetic catatonia is now defined as a neuropsychiatric syndrome principally characterised by akinesia, mutism, stupor, and catalepsy. ${ }^{1}$ Even if some advances have been made in the recognition of catatonia, in particular by the development of different rating scales, ${ }^{1}$ the pathophysiology of this syndrome is not clearly established.

A right handed 14 year old girl presented with akinetic catatonia during an episode of depression in the context of a bipolar type disorder. Her catatonic status was characterised by akinesia with brief episodic spontaneous stereotyped movements, mutism, no spontaneous oral intake, catalepsy, waxy flexibility, and stupor with brief occasional eye contacts. This corresponded to a total score of 19 on the Northoff Catatonia Scale. ${ }^{1}$ Electroencephalogram performed one day after onset of symptoms showed diffuse theta activity with sporadic diffuse delta activity. Cerebral magnetic resonance imaging was normal. Brain positron emission tomographies (PET) were obtained on a CTI-Siemens HR+ tomograph. A first PET (PETl) using $\left[{ }^{18} \mathrm{~F}\right]-$ fluorodeoxyglucose (FDG) was performed on day 2 in a drug free state. Thereafter, intramuscular injection of $2 \mathrm{mg}$ of lorazepam induced rapid clinical remission of the akinetic phase. Oral lorazepam was then given ( $3.75 \mathrm{mg} /$ day) during five days. On day 8 , a second PET with FDG was performed while the patient was treated by olanzapine ( 15 $\mathrm{mg} /$ day) and presented hyperactivity, logorrhoea, and disinhibition characterised by uncontrolled social interactions and physical contacts. Neuropsychological testing performed some days after remission revealed no apraxia or language disturbances but dysfunction of executive tasks manifested in the revised Wisconsin card sorting, the Tower of London, Stroop, and Trailmaking tests.

Voxel based analyses comparing patient's cerebral glucose metabolism with that of 29 right handed healthy controls ( 16 women and 13 men, mean age 32) were performed using Statistical Parametric Mapping (SPM99) (Wellcome Department of Cognitive Neurology, London, UK). Data from each subject were normalised to a standard stereotacti space and then smoothed with a $12 \mathrm{~mm}$ full width half maximum isotropic kernel. The
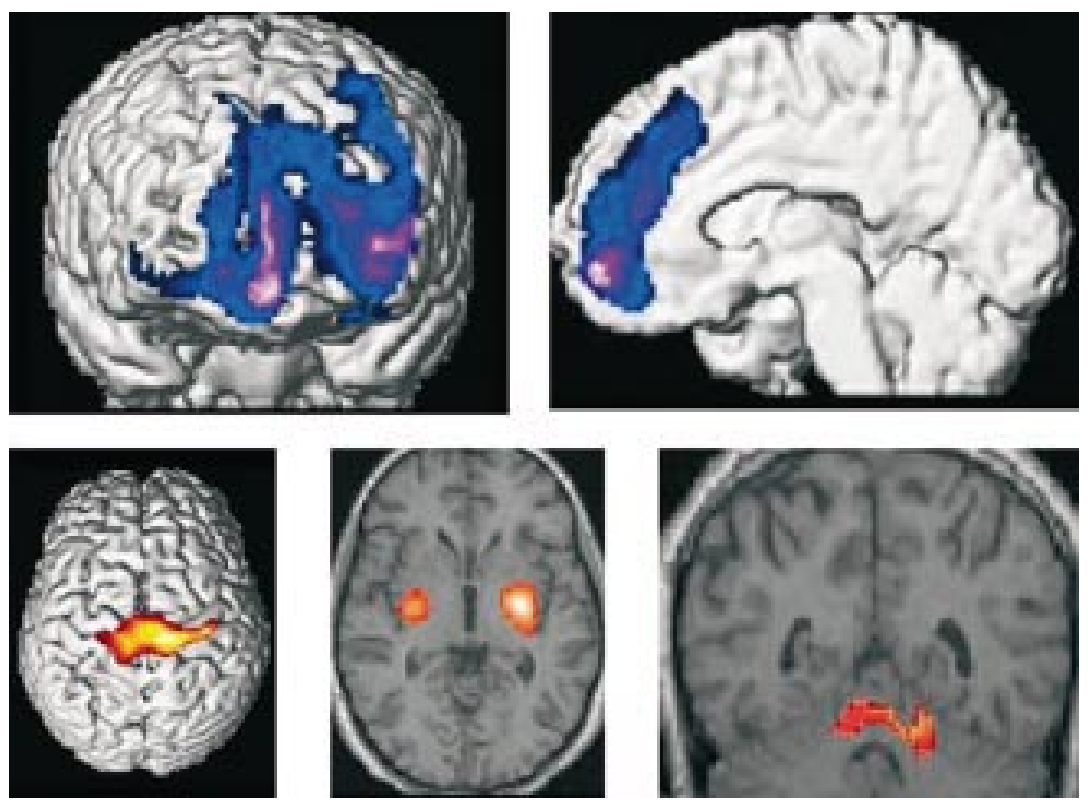

Figure 1 Results of the exclusive masking analysis showing a decrease of metabolism in a large prefrontal area (upper row, on the right), the right anterior cingulate and the right medial frontal cortices (upper row, on the left). This analysis also showed a relative increase of metabolism in primary motor cortices (lower row, on the left), in the rostral part of the striatum (lower row, in the middle), and in the vermis (lower row, on the right). Dysfunctional brain areas have been coregistrated to the patient's magnetic resonance imaging.

analysis identified brain regions where glucose metabolism was significantly changed in each patient scan compared with the control group. All results presented are significant at $\mathrm{p}<0.05$ corrected for multiple comparisons over the entire brain volume. In regions where we had a priori hypothesis-that is, regions implicated in awareness and motor control-we also considered results significant at $\mathrm{p}<0.05$ after small spherical volume correction (radius $20 \mathrm{~mm}$ ). PET2 analysis showed a relative decrease of metabolism in the precuneus, lateral parietal cortices (Brodmann area 40) and in the right superior frontal circumvolution (Brodmann area 6), see table 1. As PET2 was conducted after akinetic catatonia remission, it was used for an exclusive masking analysis of PETl in order to search for metabolic changes characteristic of the akinetic catatonic state. This showed that a large area of the prefrontal cortex (mostly on the left side) including anterior cingulate, medial prefrontal, and dorsolateral cortices presented a relative decrease of metabolism in comparison with the control group (fig 1) This analysis also revealed relative hypermetabolism of the primary motor cortex, the ros- tral part of the striatum, and the vermis (fig 1). PETl analysis also revealed that the precuneus and the left lateral parietal cortex (Brodmann area 40) presented a relative decrease of metabolism (table 1).

In our opinion, these results might shed some light on the pathogenesis of akinetic catatonia. Indeed, exclusive masking analysis allowed us to determine in this case the metabolic changes characteristic of akinetic catatonia. Prefrontal cortical areas like anterior cingulate, dorsolateral, and medial prefrontal cortices are implicated in the planning, initiation, generation of voluntary movements and executive functions in general. Hypofunction of these brain areas, as demonstrated in our patient, could therefore explain symptoms such as akinesia, mutism, and absence of spontaneous oral intake, which are usual features of akinetic catatonia. ${ }^{1}$ Moreover, the increased activity in primary motor cortices, the rostral part of the striatum and the vermis, associated with the deficit of internal initiation and generation of voluntary movements, might account for some particular motor features of catatonic states. These are

Table 1 Results of SPM analysis of PET1 and 2

\begin{tabular}{|c|c|c|c|c|c|c|c|c|c|c|}
\hline \multirow[b]{3}{*}{ PET } & \multicolumn{5}{|c|}{ Hypermetabolism } & \multicolumn{5}{|c|}{ Hypometabolism } \\
\hline & \multicolumn{2}{|c|}{ Cluster level } & \multicolumn{3}{|c|}{ Voxel level } & \multicolumn{2}{|c|}{ Cluster level } & \multicolumn{3}{|c|}{ Voxel level } \\
\hline & $\mathrm{p}$ & Cluster size & $\mathrm{p}$ & z & Coordinates $x, y, z(\mathrm{~mm})$ & $\mathrm{p}$ & Cluster size & $\mathrm{p}$ & z & Coordinates $x, y, z(\mathrm{~mm})$ \\
\hline 1 & $\begin{array}{l}0.001 \\
<0.001 \\
<0.001 \\
0.008\end{array}$ & $\begin{array}{l}885 \\
2485 \\
977 \\
549\end{array}$ & $\begin{array}{l}0.008 \\
0.024 \\
0.003^{*} \\
0.004^{*}\end{array}$ & $\begin{array}{l}4.97 \\
4.71 \\
4.27 \\
4.24\end{array}$ & $\begin{array}{l}30,-8,-2 \\
10,-24,72 \\
12,-52,-1 \\
-30,-12,-2\end{array}$ & $\begin{array}{l}0.039 \\
<0.001 \\
<0.001 \\
0.002\end{array}$ & $\begin{array}{l}354 \\
1752 \\
8720 \\
733\end{array}$ & $\begin{array}{l}0.015 \\
0.001^{*} \\
0.002^{*} \\
0.004^{*}\end{array}$ & $\begin{array}{l}4.83 \\
4.49 \\
4.40 \\
4.21\end{array}$ & $\begin{array}{l}46,18,0 \\
-4,-54,30 \\
-24,56,18 \\
-50,-60,18\end{array}$ \\
\hline 2 & & & & & & $\begin{array}{l}0.005 \\
<0.001 \\
<0.001\end{array}$ & $\begin{array}{l}628 \\
1589 \\
1057\end{array}$ & $\begin{array}{l}0.016 \\
0.002^{*} \\
0.005^{*}\end{array}$ & $\begin{array}{l}4.81 \\
4.43 \\
4.16\end{array}$ & $\begin{array}{l}40,-60,44 \\
-4,-54,30 \\
-48,-64,32\end{array}$ \\
\hline
\end{tabular}

*After small spherical volume correction (radius $20 \mathrm{~mm}$ ). 
the occurrence of episodic spontaneous stereotyped movements and the prolonged maintenance of posture (catalepsy). Previous functional cerebral imaging studies have reported the implication of the vermis in the maintenance of standing postures. ${ }^{2}$ The high metabolic activity observed in the motor cortex could be related to reduced neuronal inhibition. Indeed, reduced density of inhibitory GABA receptors in this area has been reported in catatonia. ${ }^{3}$ Previous imaging studies found dysfunctional posterior lateral parietal cortex in the catatonic state. ${ }^{4}$ PETl analysis showed hypofunction of this left region which persisted after clinical remission. So, this regional dysfunction is not sufficient to lead to akinetic catatonia but it might have participated in the disturbance of executive tasks planning.

Patients with akinetic catatonia are classically unresponsive to their environment. ${ }^{1}$ This symptom characterises the stuporous state encountered in this syndrome. The exclusive masking analysis demonstrated reduced activity in the medial prefrontal cortex during akinetic catatonia. Previous functional imaging studies showed that the ventral medial prefrontal cortex is implicated in the integration of the visceromotor aspects of emotional processing with information gathered from the internal and external environments. ${ }^{5}$ The dorsal medial prefrontal cortex has been involved in explicit representations of states of the "self". 5 Dysfunction of these brain areas might therefore explain the stuporous state observed in akinetic catatonia. Activity within the precuneus has been implicated in the representation of the world around us and the lateral parietal cortex is known to participate in conscious awareness. ${ }^{5}$ PETl analysis showed that these two regions presented a decrease of metabolism that persisted on PET2. This persistence could be related to the hypomaniac state presented at the time of PET2, a state, which differs from the resting state of the control subjects. Indeed, high level of glucose metabolism in the precuneus and lateral parietal cortex is the metabolic hallmark of the normal resting state..$^{5}$ Despite it persistence after catatonia remission, dysfunction of these regions during the akinetic catatonic state may be a prerequisite for the establishment of its stuporous aspect, as supported by studies on patients with reduced level of consciousness

In conclusion, some motor symptoms usually encountered in akinetic catatonia may be related to dysfunction of prefrontal cortical areas but also primary motor cortex, striatum, and vermis. This case of akinetic catatonia also brings new clues for the involvement of the medial prefrontal cortex in conscious awareness.

X De Tiège, $\mathrm{S}$ Laureys, $\mathrm{S}$ Goldman PET/Biomedical Cyclotron Unit, Erasme Hospital Free University of Brussels, Brussels, Belgium

$X$ De Tiège, I Massat, F Lotstra, J Mendlewicz

Department of Psychiatry, Erasme Hospital, Free University of Brussels

J-C Bier

Department of Neurology, Erasme Hospital, Free University of Brussels

J Berré

Intensive Care Unit, Erasme Hospital, Free University of Brussels
Correspondence to: $\operatorname{Dr} X$ De Tiège, PET/Biomedical Cyclotron Unit and Department of Psychiatry, Erasme Hospital, Free University of Brussels, Brussels, Belgium; xdetiege@hotmail.com

\section{References}

1 Northoff G, Koch A, Wenke J, et al. Catatonia as a psychomotor syndrome: a rating scale and extrapyramidal motor symptoms. Mov Disord 1999;14:404-16

2 Ouchi Y, Okada H, Yoshikawa E, et al. Absolute changes in regional cerebral blood flow in association with upright posture in humans: an orthostatic PET study. J Nucl Med 2001:42:707-12

3 Northoff G, Steinke R, Czcervenka C, et al. Decreased density of GABA-A receptors in the left sensorimotor cortex in akinetic catatonia: investigation of in vivo benzodiazepine receptor binding. J Neurol Neurosurg Psychiatry 1999;67:445-50

4 Northoff G, Steinke R, Nagel DC, et al. Right lower prefronto-parietal cortical dysfunction in akinetic catatonia: a combined study of neuropsychology and regional cerebral blood flow. Psychol Med 2000;30:583-96.

5 Gusnard DA, Raichle ME. Searching for a baseline: functional imaging and the resting human brain. Nat Rev Neurosci 2001;2:685-94.

\section{Mirth and laughter arising from human temporal cortex}

Laughter and mirth are essential in our enjoyment of daily life and in facilitating communication. Various studies have been done relating to the emotional processing that takes place in the human cerebral cortex, but few have explored the cerebral origins of mirth. Some reports on pathological laughter have implicated the hypothalamus, brain stem, and temporal lobe.

As part of the presurgical evaluation of patients with epilepsy, electric cortical stimulation is used to delineate the functional cortical areas, and sometimes this elicits various emotional responses. ${ }^{4}$ However, only two stimulation studies ${ }^{25}$ have been conducted with a focus on mirth and laughter. Arroyo et al suggested that the motor act of laughter and the processing of its emotional conten were separately represented in, respectively, the anterior cingulate area and the basal temporal area (the fusiform gyrus or parahippocampal gyrus, or both). ${ }^{2}$ Fried et al suggested not only that laughter and mirth were represented in the presupplementary motor area, but also that there was close linkage between the motor, affective, and cognitive components of laughter.

We report a patient in whom electric cortical stimulation applied to the inferior temporal gyrus produced mirth alone or laughter preceded by mirth, depending on the intensity of the stimulation.

\section{Case report}

A 24 year old right handed woman with medically intractable complex partial seizures underwent implantation of subdural grid electrodes on the cortical surface of the left temporal cortex and a depth electrode into the right mesial temporal cortex. Long term video/EEG monitoring with scalp electrodes done before this invasive monitoring showed late ictal lateralisation at the left anterior temporal area, and thus a right temporal onset could not completely be excluded. Magnetic resonance imaging showed high intensity and atrophy in the left anterior and right posterior hippocampus. Interictal FDG-PET (fluorodeoxyglucose positron emission tomography) showed hypometabolism in the left

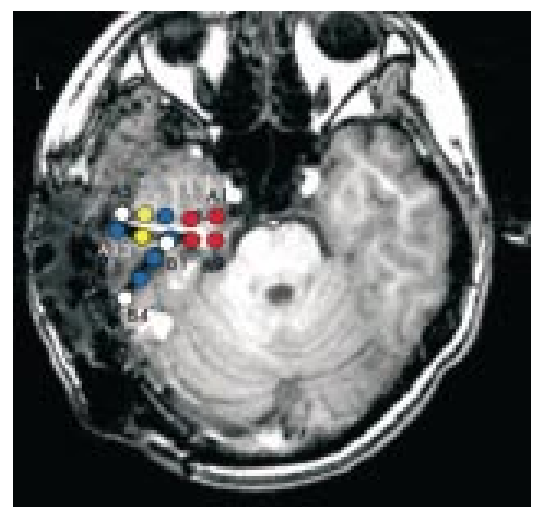

Figure 1 Brain magnetic resonance imaging showing the arrangement of electrodes on the surface of the left basal temporal cortex. A 11 and $B 1$ are overlapping. The electrodes A4 and A12 (yellow) are located on the basal aspect of inferior temporal gyrus where the electric stimulation induced mirth with or without laughter. A1, A2, A9, and A10 (red) were epileptogenic foci and were ultimately resected. Electrical stimulation of blue electrodes disrupted or arrested speech and other language tasks.

temporal lobe. An intracarotid amobarbital test revealed the dominance of language and memory in the left hemisphere.

The seizures started with an epigastric rising sensation, followed by loss of awareness combined with hand and oral automatisms. Laughter or the feeling of mirth was not seen during the patient's habitual seizures. Video/ EEG monitoring showed that the epileptogenic focus was in the left mesial temporal cortex (Al, 2, 9, and 10) (red electrodes in fig 1). Electrical cortical stimulation $(50 \mathrm{~Hz}$, alternate polarity of square pulses) was done to delineate the functional areas, with special emphasis on language function.

Stimulation of the basal aspect of the inferior temporal gyrus between A4 and Al2 (yellow electrodes in fig 1) at low intensity and short duration $(5 \mathrm{~mA}, \mathrm{l} \mathrm{s})$ consistently produced mirth without laughter, and it was always accompanied by a melody that she had heard in a television programme in her childhood. The duration and intensity of the mirth increased in proportion to the duration and intensity of stimulation ( $15 \mathrm{~mA}, 3 \mathrm{~s}$ ), and she eventually smiled during the latter part of a 5 second stimulation. The patient said that the tune appeared funny to her and made her feel amused, but only during the electrical stimulation, and we were able to confirm this. When maximum intensity at longer duration (15 $\mathrm{mA}, 5 \mathrm{~s}$ ) was applied, it disrupted a variety of language tasks, but neither alternating hand and foot movements nor vocalisation was disturbed. During this maximum stimulation condition, the patient felt mirth, but the performance of various language tasks obscured apparent laughter. Stimulation of the adjacent pairs of electrodes (A3-All, A6-A13, and B2-4) (blue electrodes in fig 1) affected only language tasks but was not consistently accompanied by a feeling of mirth.

\section{Comment}

Our observations suggest that mirth is represented in a relatively small distinct area in the temporal neocortex (the basal surface of the inferior temporal gyrus), which is in part consistent with the observations of Arroyo et al. ${ }^{2}$ 
Our study clearly showed that mirth was represented in the inferior temporal gyrus, and was closely linked with a particular context (a certain tune in this patient). This association with a specific event was not observed in the patients reported by Arroyo et al. ${ }^{2}$ Because the temporal lobe is involved in memory function in human, it is reasonable that both the context of the mirth and laughter and the induced mirth and laughter are represented. In the present case, we could not identify any site where the electric stimulation elicited laughter without mirth. Importantly, the fact that the stimulation with higher intensity and longer duration elicited mirth with laugher more effectively suggests different thresholds for mirth and laughter, postulating a hierarchical organisation or serial processing of mirth and laughter in the human temporal cortex. Laughter might be situated at a higher order than mirth, at least in the temporal neocortex. It is possible that laughter might be caused by further activation of the frontal motor cortices, including the anterior cingulate gyrus, through corticocortical projections, such that electrical cortical stimulation could elicit laughter without mirth.'

With regard to the characteristics of induced mirth in this patient, the melody which made her feel funny was not amusing by itself in the absence of electrical stimulation, raising the possibility that stimulation changed the internal standard of her amusement through an undetermined process.

Although it should be taken into account that the mirth elicited in the present case might not necessarily have reflected the representation of mirth and laughter in the normal brain, no mirth was seen during the patient's habitual seizures, and neither electrode A4 nor electrode A12 was included in the epileptogenic foci. Thus this particular area (A4-A12) producing mirth on stimulation can be judged to reflect normal function in this patient.

In the present case, mirth is represented in the temporal lobe and may be stored together with the context inducing mirth in the same area, suggesting a close relation between mirth and memory function. As far as the temporal neocortex in the present patient is concerned, laughter seems to be situated at a hierarchically higher order than mirth.
T Satow, K Usui, M Matsuhashi, J Yamamoto, T Begum, H Shibasaki Human Brain Research Centre, Kyoto University Graduate School of Medicine, 54 Kawahara-cho, Shogoin, Sakyo, Kyoto 606-8507, Japan

A lkeda

Department of Neurology, Kyoto University Graduate School of Medicine

N Mikuni, S Miyamoto, N Hashimoto Department of Neurosurgery, Kyoto University Graduate School of Medicine

Correspondence to: Dr Hiroshi Shibasaki; shib@kuhp.kyoto-u.ac.jp

\section{References}

1 Martin JP. Fits of laughter (sham mirth) in organic cerebral disease. Brain 1950;70:453-64.

2 Arroyo S, Lesser RP, Gordon B, et al. Mirth, laughter and gelastic seizure. Brain 1993;116:757-80.

3 Berkovic SF, Andermann F. Pathologic laughter. In: Joseph $A B$, Young RR, eds. Movement disorders in neurology and neuropsychology. Malden, MS: Blackwell Science, 1999:360-5

4 Penfield W, Jasper H. Epilepsy and the functional anatomy of the human brain. Boston: Little, Brown and Co, 1954:439-69.

5 Fried I, Wilson $\mathrm{CL}$, MacDonald $\mathrm{KA}$, et al. Electric current stimulates laughter. Nature 1998;391:650.

\section{How valid is the clinical diagnosis of Parkinson's disease in the community?}

In a population based study on the prevalence of Parkinson's disease in London, Schrag et al reported on the data of a long term clinical evaluation of 202 patients. ${ }^{1}$ The initial diagnosis of probable Parkinson's disease was later confirmed in $83 \%$, plus $2 \%$ each with atypical features and possible Parkinson's disease. In $15 \%$ the initial diagnosis was later rejected while $19 \%$ of patients not diagnosed as Parkinson's disease were later found to have the disorder. Their conclusion was that in 15\% of the cases the clinical criteria of Parkinson's disease were not followed, in accordance with previous retrospective clinicopathological studies of parkinsonism, in which the rate of false positive diagnosis ranged between
$22-24 \%^{23}$ and $15-18 \%{ }^{45}$ Using more strict diagnostic criteria by movement disorder experts, this figure could recently be further reduced to around $10 \%$, with a positive predictive value (PPV) for idiopathic Parkinson's disease of $98.6 \%$, and for other parkinsonian syndromes $71.4 \%$-for example, for multisystem atrophy (MSA), 85.7\%, and for progressive supranuclear palsy (PSP), $80 \%{ }^{6}$

Referring to these data, Schrag et al suggested that at least $10 \%$ of the patients with a final clinical diagnosis of Parkinson's disease may have other disorders. ${ }^{1}$ In pathological series, the incidence of atypical parkinsonism is substantial; for example, PSP is found in $6-22 \%$ of necropsy cases, MSA in $5-11.4 \%$, vascular parkinsonism in $2-3 \%$, and Alzheimer's disease in demented Parkinson's disease patients in $2-6 \%{ }^{5}$ ( see table 1 ).

Although samples from brain banks and specialised institutions are considered to overrepresent atypical disorders owing to the referral bias inherent in such samples, ${ }^{7}$ these data are, at least in part, confirmed by a large consecutive clinicopathological study of 260 elderly patients with a clinical diagnosis of parkinsonism derived in the years 1989 to 2001 from three large community hospitals in Vienna, two with acute and one with chronic care facilities (table 1). The concordance of the clinical diagnosis with the necropsy findings in this cohort was much better than in previous series ${ }^{5}$ (table 2), which, unfortunately, was not considered or quoted by Schrag et al. In our recent necropsy series, the mean incidence of Lewy body disease, including Parkinson's disease, was 78\%; of other neurodegenerative disorders masquerading as Parkinson's disease (for example, PSP, MSA, and so on), around 12\%; while other disorders referred to as secondary parkinsonism (essential tremor, drug induced parkinsonism) accounted for $8.4 \%$ (table 1 ). The initial rate of misdiagnosis in the overall group of 750 cases was around $17 \%$, and, owing to more precise diagnostic criteria, this finally fell to $11.5 \%$ (table 2).

A review of the clinical and pathological diagnoses of 160 non-demented patients with parkinsonism (85 men, 75 women; mean (SD) age, 76.6 (8.3) years, range 52 to 96) - the majority of whom had been examined in hospitals by neurologists experienced

Table 1 Incidence of different types of Parkinsonism in necropsy series (percentages)

\begin{tabular}{|c|c|c|c|c|c|c|}
\hline & \multirow[b]{2}{*}{$\begin{array}{l}\text { Schrag et al }{ }^{1} \\
\text { (clinical PD) }\end{array}$} & \multirow[b]{2}{*}{ Hughes et al } & \multirow[b]{2}{*}{$\begin{array}{l}\text { Jellinger }^{5} \\
\text { (1957-70) }\end{array}$} & \multirow{2}{*}{$\begin{array}{l}\text { Jellinger }^{5} \\
\text { (1971-88) }\end{array}$} & \multicolumn{2}{|c|}{ Jellinger (1989-2001) } \\
\hline & & & & & $\mathrm{n}$ & $\%$ \\
\hline $\begin{array}{l}\text { Idiopathic Parkinson's disease } \\
\text { (Brainstem LB disease) }\end{array}$ & $\begin{array}{l}61.4 \\
(2.0)\end{array}$ & 50.0 & 75.3 & 77.0 & 151 & 57.6 \\
\hline Lewy body dementia & & - & 2.7 & 5.8 & 53 & 20.4 \\
\hline Lewy body disease (total) & & & 78.0 & 82.8 & 204 & 78.0 \\
\hline Other degenerative parkinsonism & & 33.0 & 10.0 & 8.9 & 34 & 13.2 \\
\hline Multiple system atrophy & 1.5 & 22.0 & 4.6 & 2.3 & 9 & 3.5 \\
\hline Progressive supranuclear palsy & 3.0 & 11.0 & 3.6 & 2.6 & 8 & 3.1 \\
\hline Pick disease, corticobasal degen & - & $?$ & 0.9 & 0.5 & 2 & 0.8 \\
\hline Alzheimer's disease & - & $?$ & 0.9 & $3.5^{\mathrm{a}}$ & 15 & 5.7 \\
\hline Secondary parkinsonism & & 17.0 & 12.0 & 8.3 & 22 & 8.4 \\
\hline Vascular parkinsonism (MIE, SAE, MIX) & 5.5 & $?$ & 3.0 & 4.2 & 8 & 3.1 \\
\hline Postencephalitic parkinsonism & - & $?$ & 6.3 & 1.9 & 0 & 0 \\
\hline Symptomatic (JCD, tumours, etc) & 3.5 & $?$ & 0 & 0.3 & 3 & 1.1 \\
\hline Toxic/drug induced parkinsonism & - & $?$ & 0.9 & 0.3 & 3 & 1.1 \\
\hline Post-traumatic/boxer dementia & - & $?$ & 0.9 & 0.3 & 0 & 0 \\
\hline Unclassified/no lesion ("tremor") & 22.8 & $?$ & 0.9 & 1.3 & 8 & 3.1 \\
\hline Total & 202 & 143 & 110 & 380 & 260 & 100.0 \\
\hline
\end{tabular}

'With SN lesion 3.0.

JCD, Jakob-Creutzfeldt disease; LB, Lewy body; MIE, multi-infarct encephalopathy; MIX, Alzheimer's disease plus vascular encephalopathy; PD, Parkinson's disease; SAE, subcortical arteriosclerotic encephalopathy. 
Table 2 Misdiagnosis in necropsy series of clinical Parkinson's disease (with or without dementia)

\begin{tabular}{|c|c|c|c|c|c|c|}
\hline \multirow[b]{2}{*}{ Pathology } & \multirow{2}{*}{$\begin{array}{l}\text { Hughes et a } \\
(n=100)\end{array}$} & \multirow{2}{*}{$\begin{array}{l}\text { Raiput et a }{ }^{\beta} \\
(n=41)\end{array}$} & \multirow{2}{*}{$\begin{array}{l}\text { Jellinger } \\
(1971-88)^{5} \\
(n=380)\end{array}$} & \multicolumn{2}{|c|}{$\begin{array}{l}\text { Jellinger }(1989-2001)^{5} \\
(n=260)\end{array}$} & \multirow{2}{*}{$\begin{array}{l}\text { Hughes et al } \\
(n=143)\end{array}$} \\
\hline & & & & $\mathrm{n}$ & $\%$ & \\
\hline Alzheimer's disease & 6 & 2.0 & 2.6 & 5 & 1.9 & $?$ \\
\hline Vascular encephalopathy & 0 & 2.0 & 3.5 & 2 & 0.8 & $?$ \\
\hline Progressive supranuclear palsy & 8 & 0.0 & 1.8 & 3 & 1.1 & 3.5 \\
\hline Multiple system atrophy & 5 & 10.0 & 2.2 & 3 & 1.1 & 3.0 \\
\hline Nigral atrophy (unclassified) & 2 & 2.0 & 0.5 & 1 & 0.4 & \\
\hline MIX encephalopathy (AD+VaE) & 0 & 0.0 & 0.5 & 1 & 0.4 & \\
\hline Lewy body dementia & 1 & 0.0 & 3.6 & 12 & 4.6 & \\
\hline Pick's disease, corticobasal degeneration & 0 & 0.0 & 0.2 & 0 & 0 & 8.7 \\
\hline Normal (essential tremor?) & 1 & 0.0 & 0.3 & 2 & 0.8 & \\
\hline Others (pallido-nigral degeneration, toxic, etc) & 0 & 2.0 & 0.3 & 1 & 0.4 & \\
\hline Postencephalitic parkinsonism & 1 & 4.0 & 0 & 0 & 0 & \\
\hline Total & 24 & 22.0 & 15.3 & 30 & 11.5 & 15.2 \\
\hline
\end{tabular}

in movement disorders over a 12 year period from 1990 to the end of 2001-gave the following results: 129 were clinically diagnosed as probable idiopathic Parkinson's disease without severe dementia, and 21 as having atypical parkinsonian syndromes. The PPV of the clinical diagnosis for the whole group was $89.4 \%$ (143/160); for idiopathic Parkinson's disease, $94.2 \%$ (131/139); for PSP, only $50 \%$ (4/8); for MSA, $57.1 \%$ (4/7); and for vascular parkinsonism, $66.7 \%(4 / 6)$. The sensitivity for idiopathic Parkinson's disease was 94.2\% owing to eight false positive cases, mainly dementia with Lewy bodies (DLB), and two cases of PSP.

The diagnostic accuracy of $89.4 \%$ for the whole cohort was higher than in the group described by Hughes et al (85.3\%), ${ }^{2}$ and was similar to that of our own total group of 260 parkinsonian cases without and with dementia, where the rate of false clinical diagnosis was $11.5 \%$ (table 2). This was lower than in previous clinicopathological series from the same hospitals and the same neuropathology department (table 2).

It is of interest that the majority of cases with a false clinical diagnosis of idiopathic Parkinson's disease in our cohort had a final pathological diagnosis of DLB-mainly "pure" DLB cases which often initially present with parkinsonism. ${ }^{9}{ }^{10}$ These were not included or mentioned in either of the British series. ${ }^{1367}$ In our recent consecutive necropsy series of 260 parkinsonian cases, DLB accounted for around $20 \%$ which, owing to improved neuropathological techniques and knowledge, was much higher than in previous series (table 1). The reason for the differences between the British series and our own is a matter for debate.

The recent British studies and our own studies imply that neurologists with particular expertise in the field of movement disorders may be best at recognising the clinical syndromes of parkinsonism. However they also show clearly that neuropathological examination using modern immunohistochemical methods still represents the gold standard for the final diagnosis which, even after examination of the patients by very experienced clinicians, may differ by around $10 \%$ from the final clinical diagnosis. Improvement in the clinical consensus criteria and expertise may further reduce the rate of false clinical diagnosis of these devastating disorders - $\mathrm{a}$ possible basis for further improvements in treatment strategies.
K A Jellinger

Institute of Clinical Neurobiology, Kenyongasse 18 A-1070 Vienna, Austria

Correspondence to: Dr Kurt A Jellinger kurt.jellinger@univie.ac.a

\section{References}

1 Schrag A, Ben-Shlomo Y, Quinn N. How valid is the clinical diagnosis of Parkinson's disease in the community? J Neurol Neurosurg Psychiatry 2002:73:529-34.

2 Hughes AJ, Ben-Shlomo Y, Daniel SE, et al. What features improve the accuracy of clinical diagnosis in Parkinson's disease: a clinicopathologic study. Neurology 1992;42: 1 142-6.

3 Rajput AH, Rozdilsky B, Rajput A. Accuracy of clinical diagnosis in parkinsonism - a prospective study. Can J Neurol Sci $1991 ; 18: 275-8$

4 Ansorge O, Lees AV, Daniel SE. Update on the accuracy of clinical diagnosis of idiopathic Parkinson's disease. Mov Disord 1997;12(suppl 1):96.

5 Jellinger KA. The pathology of Parkinson's disease. Adv Neurol 2001;86:55-72.

6 Hughes AJ, Daniel SE, Lees AJ. Improved accuracy of clinical diagnosis of Lewy body Parkinson's disease. Neurology 2001:57:1497-9.

7 Hughes AJ, Daniel SE, Ben-Shlomo Y, et al. The accuracy of diagnosis of parkinsonian syndromes in a specialized movement disorder service. Brain 2002;1 25:861-70.

8 Schrag A, Ben-Shlomo Y, Quinn NP. Prevalence of progressive supranuclear palsy and multiple system atrophy: a cross-sectional study. Lancet 1999;354:1771-5.

9 McKeith IG, Galasko D, Kosaka K, et al. Consensus guidelines for the clinical and pathologic diagnosis of dementia with Lewy bodies (DLB): report of the consortium on DLB international workshop. Neurology 1996;47: 1113-24.

10 Seppi K, Wenning GK, Jellinger K, et al. Disease progression of dementia with Lewy bodies: a clinico-pathological study [abstract]. Neurology 2000;45(suppl 3):A391.

\section{Smoking and cognitive change from age 11 to age 80}

Age related cognitive decline affects people's quality of life and their ability to live independently. ${ }^{1}$ A recent review stated, "[we] are aware of no studies on the relationship between smoking and cognitive decline associated with normal aging or studies of the effect of smoking on cognition in normally aging individuals." ${ }^{\prime \prime}$ Some previous studies examined smoking in relation to pathological cognitive aging, but lacked cognitive data before the initiation of smoking, and used crude clinical cognitive assessments. ${ }^{2-4}$ Among middle aged subjects, current smoking was associated with poorer cognitive performance on tasks of psychomotor speed and cognitive flexibility. ${ }^{5}$ Smoking has been identified as a possible risk factor for accelerated cerebral degenerative changes, cognitive decline, and dementia. ${ }^{6}$ Here we show that smoking contributes to normal cognitive change from age 11 to age 80

\section{Participants, methods, and results}

The Scottish Mental Survey of 1932 (SMS1932) tested mental ability in people born in 1921 ( $\mathrm{n}=87498)$. The SMS1932's Moray House test (MHT) was validated against the Stanford Binet test and includes verbal reasoning, numerical, spatial, and other items. From 1999 to 2001 we traced and retested 550 people from Edinburgh who were born in 1921 (the Lothian birth cohort 1921). All lived independently. We excluded people with mini-mental state examination scores below 24 and those with known dementia. We traced their scores on the MHT from SMS1932, readministered the MHT using the same instructions and time limit as the SMS1932, and collected information on smoking. In all, 470 people (194 men) provided full data.

We examined the effect of smoking on cognitive change from age 11 to age 80 using general linear modelling (analysis of covariance; SPSS version 11). Age corrected MHT score at age 80 was the dependent variable, smoking (never $(\mathrm{n}=205)$; current $(\mathrm{n}=34)$; ex-smoker $(n=231))$ and sex were between subject variables, and age corrected MHT score at age 11 was a covariate. Among the current smokers the mean (SD) age at starting smoking was 18.9 (5.5) years (range 9 to 40). The ex-smokers' mean age at starting smoking was $18.2(5.2)$ years (range 7 to 60 ), and the mean age at stopping smoking was 49.6 (16.1) years (range 19 to 79 years). Only six of these ever-smokers (current and ex-) began smoking before the age of 11 . The mean (SD) MHT scores for each smoking related subgroup at age 11 and age 80 are shown in table 1 . MHT scores at age 11 had a large effect on scores at age $80\left(F_{1,463}=332.2, \mathrm{p}<0.001\right.$, $\left.\eta^{2}=0.418\right)$. There was a significant, independent effect of smoking $\left(F_{2,463}=3.3\right.$, $\left.\mathrm{p}=0.039, \quad \eta^{2}=0.014\right)$, but not of sex 
Table 1 Moray House test scores at age 11 and age 80 by smoking status

\begin{tabular}{llll}
\hline & $\mathrm{n}$ & IQ age $11(\mathrm{SD})$ & IQ age $80(\mathrm{SD})$ \\
\hline Never smoked & 205 & $101.6(13.8)$ & $100.8(14.5)$ \\
Ex-smoker & 231 & $99.8(15.2)$ & $100.3(14.1)$ \\
Current smoker & 34 & $98.4(15.5)$ & $94.3(17.5)$ \\
\hline
\end{tabular}

Scores were converted to IQ-type scores (mean $=100 ; S D=15$ ) at each age separately.

$\left(F_{1,63}=3.1, \mathrm{p}=0.079, \eta^{2}=0.007\right) .^{2}$ The sex by smoking interaction was not significant $\left(F_{2,463}=1.7, \mathrm{p}=0.17, \eta^{2}=0.007\right)$. Current smokers had significantly lower MHT scores at age 80 than never smokers $(\mathrm{p}=0.013$; mean difference $=-5.2,95 \%$ confidence interval (CI) -9.4 to -1.1 ) and ex-smokers $(\mathrm{p}=0.016$; mean difference $=-5.0,95 \% \mathrm{CI}$ -9.0 to -0.9 ). These group comparisons remained similar in effect size and significance after entering years of full time education to the model.

\section{Comment}

Smoking affects cognitive change detrimentally from age 11 to age 80 , with an effect that is similar in size to other contributors, such as the $\epsilon 4$ allele of the APOE gene. ${ }^{7}$ An advantage of this study is that the initial cognitive assessments were made when only a tiny percentage of the subjects had begun smoking. This finding adds to those of a previous study which found that, among middle aged participants, current smokers had reduced cognitive performance when compared with never smokers. ${ }^{5}$ In the present study, a history of having smoked and then given up smoking was not associated with any lowering of cognitive scores in old age. At age 80 there are survivor effects on cohorts owing to factorssuch as death and illnesses-that are related to smoking. It might be expected that smokers in our cohort would be biased toward being especially fit and cognitively able. Thus selection bias could lead to our underestimating the effect of smoking on cognitive aging. The effect of smoking on cognitive aging might be direct, associated with, for example, biochemical factors such as antioxidant defences; neuropathological changes including acceleration of perfusional decline, cerebra atrophy, and polioaraiosis and leucoaraiosis (thinning of grey and white matter densities, respectively $)^{6}$; or smoking related diseasethough smoking did not explain the effect of cardiovascular disease on cognition in the Rotterdam study, ${ }^{4}$ nor unequivocally in the Zutphen study. ${ }^{3}$ It might also be indirect, being an indicator of a general tendency toward healthy lifestyle choices and responsiveness to health education. These possibilities notwithstanding, our data add to the reasons for giving up smoking, irrespective of age

\section{Acknowledgements}

The Lothian birth cohort data were gathered with support from a Biotechnology and Biological Sciences Research Council Science of Ageing (SAGE) grant. IJD is the recipient of a Royal Society-Wolfson research merit award. LJW holds a Wellcome Trust career development award.

Competing interests: none declared
I J Deary, A Pattie, M D Taylor, M C Whiteman

Psychology, School of Philosophy, Psychology and Language Sciences, University of Edinburgh, 7 George Square, Edinburgh EH8 9JZ, Scotland,

J M Starr

Department of Geriatric Medicine, University of Edinburgh

L J Whalley

Department of Mental Health, University of Aberdeen, Aberdeen, Scotland, UK

Correspondence to: Professor Deary; i.deary@ed.ac.uk

\section{References}

1 Fillit HM, Butler RN, O'Connell AW, et al. Achieving and maintaining cognitive vitality with aging. Mayo Clin Proc 2002;77:681-96.

2 Cervilla JA, Prince M, Mann A. Smoking, drinking, and incident cognitive impairment: a cohort community based study included in the Gospel Oak project. J Neurol Neurosurg Psychiatry 2000;68:622-6.

3 Launer LJ, Feskens EJM, Kalmijn S, et al. Smoking, drinking and thinking: the Zutphen Elderly Study. Am J Epidemiol 1996;143:219-27.

4 Breteler MMB, Claus JJ, Grobbee DE, et al. Cardiovascular disease and distribution of cognitive function in elderly people: the Rotterdam study. BM 1994;308:1604-8.

5 Kalmijn S, van Boxtel MPJ, Verschuren MWM, et al. Cigarette smoking and alcohol consumption in relation to cognitive performance in middle age. Am J Epidemiol 2002; 156:936-44

6 Meyer JS, Rauch GM, Crawford K, et al. Risk factors accelerating cerebral degenerative changes, cognitive decline and dementia. Int J Geriatr Psychiatry 1999;14:1050-61.

7 Deary IJ, Whiteman MC, Pattie A, et al. Cognitive change and the APOE $\epsilon 4$ allele. Nature 2002;418:932. 


\section{CORRESPONDENCE}

\section{Neutralising antibodies to interferon $\beta$ during the treatment of multiple sclerosis}

Giovannoni and colleagues are to be commended for their detailed analysis of the impact of neutralising antibodies (NAB) to interferon $\beta$ (IFN $\beta$ ) during the treatment of multiple sclerosis. ${ }^{1}$ We are in general agreement with many of their statements and conclusions, but a few points should be discussed in a wider context.

With respect to the clinical significance of neutralising antibodies to IFN $\beta$, the authors state that "IFN $\beta$ has little if any clinical and MRI efficacy in the presence of neutralising antibodies." We think it is appropriate to be more circumspect, as most published studies suggest that in $\mathrm{NAB}$ positive patients, clinical (and MRI) efficacy of interferon treatment is present when compared to placebo, and that there is some evidence that more immunogenic higher dose treatment can be more effective than less immunogenic lower dose treatment. ${ }^{2}$ Giovannoni et al appear to base their statement on the increase in T2 burden of disease in the NAB positive group in the PRISMS extension study, but they do no mention similar comparisons which, if interpreted in the same way, would indicate that the NAB positive group does better than the placebo group. ${ }^{3}$ For example, the relapse rate in placebo patients was 1.3 year in years one to two, whereas it was 0.81 and 0.50 in NAB positive and $\mathrm{NAB}$ negative high dose patients in years three to four. We recognise that this specific comparison is fraught with difficulties owing to time trends in the relapse data, but these potential difficulties are present in all such comparisons. In a recent paper we report-in probably the largest study of neutralising antibodies in multiple sclerosis, describing $100 \mathrm{NAB}$ positive patients in the European SPMS study-that high titres of neutralising antibodies do have a clinical impact, but that this impact is rather limited, and that on both clinical and MRI measures patients on active treatment who develop neutralising antibodies continue to do consistently better than those on placebo. ${ }^{4}$ The main conclusions of this paper are based on longitudinal analyses of the data on those patients who switched from NAB negative to $\mathrm{NAB}$ positive status; this is the only statistical approach that allows a direct assessment of whether the change from $\mathrm{NAB}$ negative to $\mathrm{NAB}$ positive status is associated with diminished efficacy of a treatment. Cross sectional comparisons are not fully reliable for establishing the impact of neutralising antibody positivity, as NAB positive and negative subgroups may differ on baseline variables (maybe unobserved) that are predictive of both neutralising antibody formation and diminished clinical response.

Giovannoni et al also state that during continued treatment "in the case of IFN $\beta-\mathrm{Ib}$ some $\mathrm{NAB}$ positive patients revert to $\mathrm{NAB}$ negative status over two to five years of follow up" and that "patients with high titres of neutralising antibodies seldom revert to being negative." In the European study of IFN $\beta$ - $1 \mathrm{~b}$ in secondary progressive multiple sclerosis the proportion of treated patients who have been $\mathrm{NAB}$ positive and subsequently revert back to being $\mathrm{NAB}$ negative is about $40 \%$ after a treatment duration up to three years (without convincing evidence that patients with higher titres revert less frequently), whereas in the study by Rice et al this percentage is close to $80 \%$ after a mean treatment duration of more than eight years. ${ }^{4}$

In our opinion, these data suggest that the clinical impact of neutralising antibodies to IFN $\beta$ during the treatment of multiple sclerosis may be more limited and more transient than suggested in the editorial, and that the development of neutralising antibodies in itself does not provide justification for switching treatments or for considering (aggressive) strategies to reduce or revert the development of neutralising antibodies. Given the current rather uncertain state of knowledge concerning the impact of neutralising antibodies, we advocate that treatment decisions should be based on clinical grounds rather than on neutralising antibody titres.

C H Polman

Department of Neurology, VU Medical Centre, 1007 MB Amsterdam, Netherlands

L Kappos

Department of Neurology, University Hospitals Basel, Switzerland

J Petkau

Department of Statistics, University of British Columbia, Vancouver, Canada

A Thompson

Institute of Neurology, University College London,

Correspondence to: Professor C H Polman ch.polman@vumc.n

\section{References}

Giovannoni G, Munschaver FE, Deisenhammer $F$. Neutralising antibodies to interferon $\beta$ during treatment of multiple sclerosis. J Neurol Neurosurg Psychiatry 2002;73:465-9.

2 Durelli L, Verdun E, Barbero $\mathrm{P}$, et al. Every-other-day interferon beta- $1 \mathrm{~b}$ versus once-weekly interferon beta- $1^{\circ}$ for multiple sclerosis: results of a 2-year prospective randomised multicentre study (INCOMIN) lancet 2002;359:1453-60.

3 PRISMS-4. Long-term efficacy of interferon beta-la in relapsing MS. Neurology 2001;56:1628-36

4 Polman C, Kappos L, White R, et al. Neutralizing antibodies during treatment of secondary progressive MS with interferon beta-1b. Neurology 2003;60:37-43.

5 Rice GP, Paszner B, Oger J, et al. The evolution of neutralizing antibodies in multiple sclerosis patients treated with interferon beta-1 b. Neurology 1999;52:1277-9.

\section{Neutralising antibodies to interferon $\beta$}

I read the editorial by Dr G Giovannoni and colleagues ${ }^{1}$ with great interest. I have, however, to report a minor error concerning the list of the excipients of the Rebif reported in their table 1. In the table the authors reported the following excipients: mannitol, HSA, sodium acetate, acetic acid, sodium chloride. Actually, as you can check in the summary of product characteristics published from EMEA (www.emea.eu.int) on 29 March 1999, in the list of excipients sodium chloride is absent, whereas sodium hydroxide is present.

C Ortenzi

Department of Molecular, Cellular and Animal Biology, University of Camerino, 62032 Camerino, Italy; claudio.ortenzi@tin.it

\section{Reference}

1 Giovannoni G, Munschaver FE, Deisenhammer F. Neutralising antibodies to interferon $\beta$ during treatment of multiple sclerosis. J Neurol Neurosurg Psychiatry 2002:73:465-9.

\section{Authors' reply}

We would like to thank Dr Ortenzi for pointing out our transcription error in relation to the excipients of Rebif ${ }^{\circledR}$ in table 1 of our

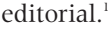

We agree with Polman and colleagues that recent comparisons show that the more immunogenic higher dose interferon $\beta$ (IFN $\beta$ ) preparations are more efficacious than the lower dose less immunogenic preparations over 24 month $^{2}$ and six month ${ }^{3}$ periods of observation. However, as discussed in our editorial, the development of neutralising antibodies and their effects on the clinical efficacy of IFN $\beta$ are delayed. In the PRISMS study the effect of neutralising antibodies on clinical efficacy only became apparent in years 3-4. In the pivotal IFN $\beta$-lb study an effect on relapse rate was only observed in the 19-24 and 25-30 month epochs. ${ }^{5}$ Hence we would argue that these comparative studies ${ }^{2}{ }^{3}$ are simply too short, and in the case of the INCOMIN trial underpowered $(\mathrm{n}=188),{ }^{2}$ to demonstrate an effect of neutralising antibodies on clinical efficacy. It is therefore impossible to extrapolate the significant short term differences shown in these studies beyond the periods of observation reported.

Because of regression to mean and the well documented tendency for the relapse rate to decrease with disease duration, it is not possible to draw any meaningful conclusions from a comparison of the relapse rate in years $1-2$ and years 3-4 from the PRISMS extension study ${ }^{46}$ In addition to the impact of neutralising antibodies on relapse rate, the PRISMS extension study clearly shows-using the more objective T2 lesion volume or burden of disease-that the average annualised increase in lesion volume over four years in the neutralising antibody positive $(\mathrm{NAB}+)$ patients is similar to the increase in the annualised lesion volume in the placebo treated patients in the first two years of the study $(\mathrm{NAB}+4.4 \% v$ placebo $5.45 \%) .{ }^{46}$ Similarly, in the IFN $\beta$-lb study, ${ }^{5}$ the annualised relapse rate of $\mathrm{NAB}+$ patients is identical to patients on placebo $(1.08 v 1.06)$. In the IFN $\beta-1 a$ (Avonex ${ }^{\circledR}$ ) trial, ${ }^{7}$ the impact of neutralising antibodies was limited to MRI outcomes. The failure of neutralising antibodies to have an effect on disease progression and relapse rate in this study probably reflects the size and duration of follow $\mathrm{u}$, as the study was terminated prematurely. It is these data from the pivotal relapsing multiple sclerosis clinical 
trials, and other studies on in vivo markers of IFN $\beta$ activity discussed in our editorial, that we use to support our statement that "interferon $\beta$ has little if any clinical and MRI efficacy in the presence of neutralising antibodies."

Data on the impact of neutralising antibodies in secondary progressive multiple sclerosis (SPMS) trials is less clear. This is to be expected, however, as the efficacy of IFN $\beta$ on disease progression-the primary outcome measure in SPMS trials-is limited and hence it would be difficult to demonstrate a significant impact on neutralising antibodies on the primary outcome measure when the actual therapeutic intervention itself is only marginally effective. ${ }^{89}$ It would be very surprising if neutralising antibodies had a significant impact on disease progression, as none of the trials is powered to detect an effect of neutralising antibodies on this outcome. For example, in the European SPMS study, 100/360 $(28 \%)$ of IFN $\beta-1 b$ treated patients became $\mathrm{NAB}+$ (titre $>20$ ) over the course of the trial. $^{10}$ Taking a conservative approach by applying the results from the trial, ${ }^{810}$ and assuming that $\mathrm{NAB}+$ patients behave as if they are on placebo and $\mathrm{NAB}$ - patients behave like the original IFN $\beta$-lb treated cohort, one would expect $49.8 \%$ of the $100 \mathrm{NAB}+$ patients to progress over three years, compared with $38.9 \%$ of the $260 \mathrm{NAB}-$ patients. At the same level of significance (0.029) from the original study, a two sided test would only have a 35\% chance of detecting a significant difference between $\mathrm{NAB}+$ and $\mathrm{NAB}-$ patients (Fisher's exact test). Compare this to a power of $80 \%$ used in the design of the original study. This power calculation is an overestimate as it ignores the therapeutic effect observed before the development of neutralising antibodies, as evidenced in this study, ${ }^{10}$ which if taken into account has the potential to further reduce the power of the subanalysis. Polman and colleagues further reduce the power of the subanalysis by limiting the longitudinal study to "switchers" - that is, clinical responses are compared within individual patients during $\mathrm{NAB}-$ and $\mathrm{NAB}+$ periods. $^{10}$ This longitudinal approach reduces the number of patients available for analysis and potentially shortens the period of observation. A longitudinal approach would seem reasonable if there are no carryover therapeutic effects of IFN $\beta$-lb treatment from the $\mathrm{NAB}-$ to $\mathrm{NAB}+$ phase and if the follow up in the $\mathrm{NAB}+$ phase is of suffi cient duration to account for the delayed effects (24 to 48 months) of neutralising antibodies on clinical efficacy. In this study the mean follow up in the $\mathrm{NAB}+$ phase would be on average too short (less than 24 months) for one to be confident of excluding a delayed effect of neutralising antibodies on disease progression. Despite the lack of power of these subanalyses, they produce some surprising results. In the cross sectional study there was a trend towards greater disease activity in the $\mathrm{NAB}+$ group in the third year, and a significant percentage $\mathrm{T} 2$ volume change from baseline to year 1 , year 2 , and the last visit ${ }^{10}$; in the underpowered and potentially flawed longitudinal analysis there was no indication of an attenuation of treatment effects on disability progression but, surprisingly considering the lower relapse rate in secondary progressive multiple sclerosis, there was a robust effect on relapse rate. ${ }^{10}$

Another way of interpreting the European SPMS NAB data as presented by Polman and colleagues is that the much higher dose of IFN $\beta$-lb ( $875 \mu \mathrm{g} /$ week) given in that study, in comparison with the lower licensed doses of
IFN $\beta$-la (30-132 $\mu \mathrm{g} /$ week), acted to quench some of the neutralising activity of the antibodies. ${ }^{10}$ Similarly, the higher doses may be responsible for inducing high dose tolerance in a subset of the patients. These phenomena are well observed with other biologicals in which the read-outs are more objective than in multiple sclerosis-for example, coagulation in anti-factor VIII and glucose levels in anti-insulin antibody positive patients.

Polman and colleagues have misinterpreted our recommendations.' We do not recommend routine screening of neutralising antibodies at present, nor the switching of treatments in $\mathrm{NAB}+$ patients unless clinically justified, nor aggressive strategies to reduce or reverse the development of neutralising antibodies. ${ }^{1}$ We simply state that further research is necessary to assess whether these strategies are appropriate. Polman and colleagues' concluding statement that treatment decisions should be based on clinical grounds rather than on neutralising antibody titres is entirely in keeping with our recommendations.

We disagree with Polman and colleagues' statement that "the clinical impact of neutralising antibodies to interferon $\beta$ during treatment of multiple sclerosis may be more limited and more transient than suggested in the editorial." Short to intermediate term data $(<4$ years $)$ from the relapsing multiple sclerosis studies discussed above ${ }^{47}$ do not support this claim, and long term clinical data (>4 years) on the effects of transient neutralising antibodies on the therapeutic efficacy of IFN $\beta$ - lb do not exist to support the latter half of their claim. In addition, evidence is yet to surface on whether or not the phenomenon of transient high titre neutralising antibodies occurs to a similar degree in patients treated with IFN $\beta$-la; therefore the latter half of their statement, if true, may not be applicable to patients treated with IFN $\beta$ la.

In conclusion, clinicians cannot ignore the issue of neutralising antibodies, particularly in view of the evidence from other fields of medicine in which neutralising antibodies reduce or inhibit the efficacy of a wide range of biologicals, including type I interferons. Why should interferon treatment in multiple sclerosis be any different?

G Giovannoni

Department of Neuroinflammation, Institute of Neurology, Queen Square, London WC1 3BG, UK

F Deisenhammer

Department of Neurology, University of Innsbruck, Innsbruck, Austria

F E Munschauer

William C Baird Multiple Sclerosis Research Center, State University of New York, Buffalo, USA

Correspondence to: Dr Gavin Giovannoni; g.giovannoni@ion.ucl.ac.uk

\section{References}

1 Giovannoni G, Munschauer FE, Deisenhammer F. Neutralising antibodies to interferon beta during the treatment of multiple sclerosis. J Neurol Neurosurg Psychiatry 2002;73:465-9.

2 Durelli L, Verdun E, Barbero $P$, et al. Every-other-day interferon beta- $1 \mathrm{~b}$ versus once-weekly interferon beta-la for multiple sclerosis: results of a 2-year prospective randomised multicentre study (INCOMIN). Lancet 2002;359: 1453-60.

3 Panitch H, Goodin DS, Francis G, et al. Randomized, comparative study of interferon beta-la treatment regimens in MS: the
EVIDENCE trial. Neurology

2002;59:1496-506

4 PRISMS-4. Long-term efficacy of

interferon-beta-la in relapsing MS. Neurology

2001;56:1628-36.

5 Study Groups. Neutralizing antibodies during treatment of multiple sclerosis with interferon beta-1 b: experience during the first three years. The IFNB Multiple Sclerosis Study Group and the University of British Columbia MS/MRI Analysis Group. Neurology 1996:47:889-94.

6 Study Group. Randomised double-blind placebo-controlled study of interferon beta-la in relapsing/remitting multiple sclerosis. PRISMS (prevention of relapses and disability by interferon beta-1 a subcutaneously in multiple sclerosis) Study Group. Lancet 1998;352:1498-504.

7 Jacobs LD, Cookfair DL, Rudick RA, et al. Intramuscular interferon beta-la for disease progression in relapsing multiple sclerosis. The Multiple Sclerosis Collaborative Research Group (MSCRG). Ann Neurol 1996;39:285-94.

8 European Study Group. Placebo-controlled multicentre randomised trial of interferon beta- $1 \mathrm{~b}$ in treatment of secondary progressive multiple sclerosis. European Study Group on interferon beta- $1 \mathrm{~b}$ in secondary progressive MS. Lancet 1998;352:1491-7.

9 SPECTRIMS. Secondary progressive efficacy clinical trial of recombinant interferon-beta-la in MS (SPECTRIMS) study group. Randomized controlled trial of interferon-beta-la in secondary progressive MS: clinical results. Neurology 2001;56: 1496-504.

10 Polman C, Kappos L, White R, et al. Neutralizing antibodies during treatment of secondary progressive MS with interferon $\beta-1 b$. Neurology 2003;60:37-43.

\section{A 1908 systematic review of the laterality of hysterical hemiplegia}

Since the publication of our systematic review of the laterality of functional or medically unexplained weakness and sensory disturbance (1965-2000) we have come across a study from 1908 with a similar aim.

Ernest Jones, later an eminent figure in the psychoanalytic movement, published his paper in French while working as an assistant physician at the London School of Medicine. ${ }^{2}$ He reported on the cumulative analysis of 277 cases of hysterical hemiplegia described by 146 authors in 164 articles published between 1880 and 1908. Most of this material is in French and German and includes cases mentioned in doctoral theses and books.

There was no excess of left sided hemiplegia compared with right in hysteria in his analysis- $54 \%$ had paralysis on the right side and $46 \%$ on the left. This was contrary to the prevailing opinion of the time ${ }^{34}$ and also disagrees with another less systematic review of older studies (covering 100 subjects, 13 publications and 6 authors between 1885-1937).

Jones' conclusions - that the laterality of hysterical hemiplegia has no diagnostic value-were the same as ours. His study has not been cited for at least 40 years (and probably much longer even than that). It has been neglected, like many other negative studies before and since, but it deserves recognition on this subject.

J Stone, C Warlow

Division of Clinical Neurosciences, School of Molecular and Clinical Medicine, Western General Hospital, Crewe Road, Edinburgh EH4 2XU, UK 
A Carson, M Sharpe

Division of Psychiatry, School of Molecular and Clinical Medicine, University of Edinburgh, Royal Edinburgh Hospital, Morningside Park, Edinburgh EHIO 5HF, UK

Correspondence to Dr Stone istone@skull.dcn.ed.ac.uk

\section{References}

1 Stone J, Sharpe M, Carson A, et al. Are functional motor and sensory symptoms really more frequent on the left? A systematic review. I Neurol Neurosurg Psychiatry 2002;73:578-81.

2 Jones $\mathrm{E}$. Le côté affecté par l'hémiplégie hystérique. Rev Neurol 1908;16:193-6.

3 Gowers WR. Hysteria. In: A Manual of diseases of the Nervous System. London: Churchill 1892: 903-60.

4 Briquet $\mathbf{P}$. Traité clinique et thérapeutique de I'Hysterie. Paris: J.B.Ballière, 1859

5 Ley RG. An archival examination of an asymmetry of hysterical conversion symptons. J Clin Neuropsychol 1980;2.

\section{Resolution of psychiatric symptoms secondary to herpes simplex encephalitis}

We read with interest the editorial by Kennedy et al, ${ }^{1}$ detailing the short-term treatment of herpes simplex encephalitis (HSE). We agree with the authors that we canno overemphasise the seriousness of the neuropsychiatric symptoms that a number of these patients display in the long term.

We report a 55 year old woman who was diagnosed with HSE; diagnosis was confirmed with a positive PCR test for herpes simplex in the CSF and acyclovir was started the following day after presentation. After a few weeks the patient's recovery was almost complete and she was discharged home. Six months later, there was an abrupt change when the patient developed insomnia and would sit up all night watching children's videos; she also became hostile and confused She was admitted to a psychiatric unit where she continued to be confused and agitated with episodes of extreme behaviour such as undressing or trying to attack staff.

MRI showed appearances consistent with severe encephalomalacia of the right tempora lobe with evidence of gliosis in the frontal and temporal lobes consistent with previous HSE. It was surprising that the EEG tracing was normal with no focal or epileptiform features.

The patient remained in the psychiatric unit for seven months during which time she failed to respond to different antipsychotic medications and she was heavily sedated. The nursing staff reported that the patient was generally confused but there were distinctive episodes where the patient would stare and then display abusive and disruptive behaviour for periods of up to an hour once or twice a day. Carbamezepine was started and when the patient reached a dose of $400 \mathrm{mg}$ twice daily these episodes ceased completely and the patient's behaviour showed dramatic improvement. She continued to have mild cognitive impairment affecting mainly shortterm memory.

Psychiatric problems after HSE are not uncommon; Hokkanen et al found that psychiatric problems are the main cause of long term disability in these patients. ${ }^{2}$ Despite the fact that clinical relapse of HSE is well documented, ${ }^{3}$ cognitive and psychiatric problems are usually already in place in the acute stage and further deterioration or relapse is uncommon. ${ }^{2}$ In our case the comparatively long period between recovery and onset of behavioural and psychiatric symptoms seemed to cast doubt about the association with the HSE and uncertainty regarding the appropriate treatment

Vallini et al reported successful treatment of a HSE patient presenting with severe emotional liability and explosive emotional outbursts. ${ }^{4}$ The patient responded to carbamezepine, which was started after his EEG showed seizure activity detected in temporal structures. Despite the absence of any EEG abnormalities in our case, it showed a similar favourable response to carbamezepine. We feel that any patient with intermittent behavioural or psychiatric symptoms after HSE should have a therapeutic trial of carbamazepine, even in the absence of any clinical or neurophysiological evidence of seizure activity.

T A-Z K Gaber, M Eshiett

Intermediate Rehabilitation Unit, Leigh Infirmary, Greater Manchester, UK

Correspondence to: Dr T Gaber; t_gaber@mailcity.com

\section{References}

1 Kennedy PGE, Chaudhuri A. Herpes simplex encephalitis. J Neurol Neurosurg Psychiatry 2002;73:237-8.

2 Hokanen L, Launes J. Cognitive recovery instead of decline after acute encephalitis: a prospective follow up study. J Neurol Neurosurg Psychiatry 1997:63:222-7.

3 Dennett C, Klapper PE, Cleator GM. Polymerase chain reaction in the investigation of 'relapse' following herpes simplex encephalitis. J Med Virol 1996;48:129-32.

4 Vallini AD, Burns RL. Carbamezepine as therapy for psychiatric sequel of herpes simplex encephalitis. South Med J 1987;80:1590-2.

\section{Authors' reply}

Gaber and Eshiett report an interesting case of carbamazepine responsive neuropsychiatric syndrome after herpes simplex encephalitis (HSE). Neuropsychiatric symptoms after HSE are well recognised. ${ }^{1}$ The frontotemporal and limbic lesions in HSE are particularly likely to cause behavioural and psychiatric symptoms. Retrospective studies have previously implicated HSE in the delayed syndromes of violen psychoses $^{2}$ and major depression. ${ }^{3}$ However psychiatric disorders are also common after non-herpes virus encephalitis. Hunter and others had emphasised the importance of considering encephalitic antecedents, even if clinically unapparent, in the differential diagnosis of psychiatric patients. ${ }^{4}$ Long term follow up data from the National Childhood Encephalopathy study have shown more recently that $20 \%$ of the affected children developed epilepsy and a similar proportion had behavioural problems, hyperactivity or unsociable behaviour.

Besides being a first line antiepileptic, carbamazepine is also recognised to possess considerable therapeutic value in certain psychoses and is an effective long term treatment for bipolar disorder in some cases. ${ }^{6}$ Carbamazepine responsiveness in this particular case may not, therefore, imply that the psychiatric symptoms were epileptic in origin. However, EEG signatures of epilepsy are often absent interictally, and the presence of psychoses is known to normalise EEG changes ("forced normalisation") in epilepsy patients. ${ }^{7}$ In this particular case, we certainly concur with the authors' use of carbamazepine and were delighted to learn of the favourable response.
P G E Kennedy, A Chaudhuri

Division of Clinical Neurosciences, University Department of Neurology, Ground Floor, Neurology Block, Institute of Neurological Sciences, Southern General Hospital, Glasgow G5 1 4TF, UK

Correspondence to: Professor P G E Kennedy; P.G.Kennedy@clinmed.gla.ac.uk

\section{References}

1 Kennedy PGE, Chaudhuri A. Herpres simplex encephalitis. J Neurol Neurosurg Psychiatry 2002;73:237-8.

2 Cleobury JF, Skinner GRB, Thouless ME, et al. Association between psychopathic disorder and serum antibody to herpes simplex virus (type 1). BM 1971;1:438-9.

3 Lycke E, Norry R, Roos B. A serological study on mentally ill patients with particular reference to the prevalence of herpes virus infections. Br J Psychiatry 1974;124:273-9.

4 Hunter $\mathbf{R}$, Jones M, Malleson A. Abnormal cerebrospinal fluid total protein and gamma globulin levels in 256 patients admitted to a psychiatric unit. J Neurol Sci 1969;9:1 1-38.

5 Madge N, Diamond J, Miller D, et al. The National Childhood Encephalopathy study: a 10 year follow up. A report on the medical, social, behavioural and emotional outcomes after serious, acute, neurological illness in early childhood. Dev Med Child Neurol 1993;35 (suppl 68):1-117.

6 Muller-Oerlinghausen B, Berghofer A Baver M. Bipolar disorder. Lancet 2002;359 241-7.

7 Landolt H. Serial electroencephalographic investigations during psychotic episodes in epileptic patients and during schizophrenic attacks. In: Lorentz de Haas AM, ed. Lectures on epilepsy. Amsterdam: Elsevier,

1958:91-133.

\section{Radiofrequency neurotomy}

In reading the study by Govind and colleagues, ${ }^{1}$ in which they report the findings of an unblinded, uncontrolled, nonrandomised trial of radiofrequency neurotomy for the treatment of third occipital headache, we are surprised that the authors advocate this therapy.

The last statement of the abstract is: "No other form of treatment has been validated for this common form of headache". This implies that Govind et al believe they have validated radiofrequency neurotomy as a form of treatment of third occipital headache. Presumably they are prepared, given the apparently impressive numbers of responders, to forego the usual practice of placebo controlled trial.

We do not understand how the authors can expect this treatment to be realistically adopted in clinical practice with no attempt to validate it the way treatments are meant to be validated, through randomised, placebo controlled trials. The statement in their final paragraph that "some practitioners may be averse to implementing a treatment that requires repetition" could perhaps more appropriately state that "some practitioners may be averse to implementing a treatment that remains unvalidated".

The authors state that one reason they did not do a placebo controlled study is that a previous study has already validated this technique in other patients. ${ }^{2}$ That a single trial of radiofrequency neurotomy in 24 so-called "whiplash patients" is sufficient basis for the current authors to abandon validation with traditional methods seems absurd, especially when closer inspection of that trial lays it in a less positive light. ${ }^{3}$ We do not accept an argument that it was impossible to blind these subjects. It would be entirely reasonable to see just how often a placebo procedure does indeed 
"fool" the patient. Govind et al seem to have already decided that this is not possible, a convenient assumption.

Further, we are concerned that Govind et al state categorically that "among patients with whiplash injuries, third occipital headache is common". The study group from which they determine this prevalence has been reviewed elsewhere, and is wholly inappropriate for a prevalence estimate, being best described as an unusual, highly select, and heterogeneous group of subjects. ${ }^{3}$

It is of note that, in regard to validated therapies for whiplash patients, the current study would have been rejected by the criteria of the Quebec Task Force on Whiplash Associated Disorders. ${ }^{4}$ We suggest that an invasive procedure should not be advocated until it has been subjected to proper study. Fortunately, we are aware that others are undertaking properly controlled trial of this form of therapy.

O Kwan, J Friel

Correspondence to: $\mathrm{Dr} O \mathrm{Kwan}, 207,10708-97$ Street, Edmonton, Alberta, Canada T5H $2 \mathrm{~L} 8$ oliverkwan@shaw.ca

\section{References}

1 Govind J, King W, Bailey B, et al. Radiofrequency neurotomy for the treatment of third occipital headache. J Neurol Neurosurg Psychiatry 2003:74:88-93

2 Lord SM, Barnsley L, Wallis BJ, et al. Percutaneous radio-frequency neurotomy for chronic cervical zygapophysial-joint pain. N Engl J Med 1996;335:1721-6.

3 Kwan O. Friel J. Critical appraisal of facet joints injections for chronic whiplash. Med Sci Monit 2002; 8:RA191-5.

4 Spitzer WO, Skovron ML, Salmi LR, et al. Scientific Monograph of the Quebec Task Force on Whiplash Associated Disorders: redefining "whiplash" and its management Spine 1995;20(suppl 8): 1S-73S.

\section{Authors' reply}

Our study reported an audit of outcomes for a treatment of a condition for which there is no other treatment available. It showed what proportion of patients obtained complete relief of pain, and for how long. Readers who wish to adopt this treatment for their patients can do so. If not, they should explain to their patients that they, personally, cannot offer them any treatment that is known to work; but they should not claim that there is no treatment. Our study shows that there is an option.

A placebo controlled trial would not prove that this treatment does not work. The outcomes should be the same as the benchmark established by our study, unless the operators perform the procedure poorly. A placebo controlled study could only show that all or part of the outcome is attributable to non-specific effects.

We consider this to be an unlikely outcome for we have never encountered in any of our own studies, nor in the literature, results showing that $86 \%$ of patients obtain complete relief of spinal pain following a sham procedure. Radiofrequency neurotomy has been shown to be associated with placebo responses in only a small proportion of patients, and for a limited duration. ${ }^{1}$ They claim that responses to third occipital neurotomy is only a conjecture. In principle it is worthy of testing, but in practice it cannot be tested.
The precepts of informed consent require that participants in a randomised controlled be informed of all the consequences and potential complications of a procedure. Numbness in the territory of the third occipital is an unavoidable side effect of third occipital neurotomy. It is a sign that the target nerve has been coagulated. It is an essential requirement for the procedure to work. The numbness lasts as long as the pain relief lasts. In a double blind trial this side effect cannot be masked. Therefore, patients who underwent a sham procedure would automatically know that they did not have the real treatment. Thereby the patients would be unblinded. Any placebo controlled trial which suffered unblinding would be fatally flawed and, therefore, unacceptable.

Any study that used a control short of a sham procedure would also be flawed, and would not escape criticism. Pundits would argue that patients would recognise that simply blocking the nerve, or simply inserting the electrode without mimicking the two hour procedure assiduously, is an obvious sham, and that any patient so treated would exhibit a nocebo effect.

For these reasons we did not venture to conduct a placebo controlled trial. If Dr Kwan and Dr Friel can show that a sham procedure on the third occipital nerve succeeds in achieving complete relief of pain in $86 \%$ of their patients we will gladly convert to their sham procedure.

We recognise it as a pity that our study would not be accepted by systematic reviews; but that is a problem for those who rely on reviews as the only source of evidence. In that regard we stand in good company. Were we to rely only on systematic reviews, radiofrequency neurotomy for trigeminal neuralgia would not be an accepted treatment; nor would we be allowed to perform appendicectomies.

While others are satisfied to deny care to patients while they engage in purist debates about levels of evidence, we are rewarded with patients grateful for the relief that they obtain, and who report: "you must repeat the procedure because I am never going back to suffering headaches again". If someone devises a better treatment for third occipital headache, we will adopt it. In the meantime

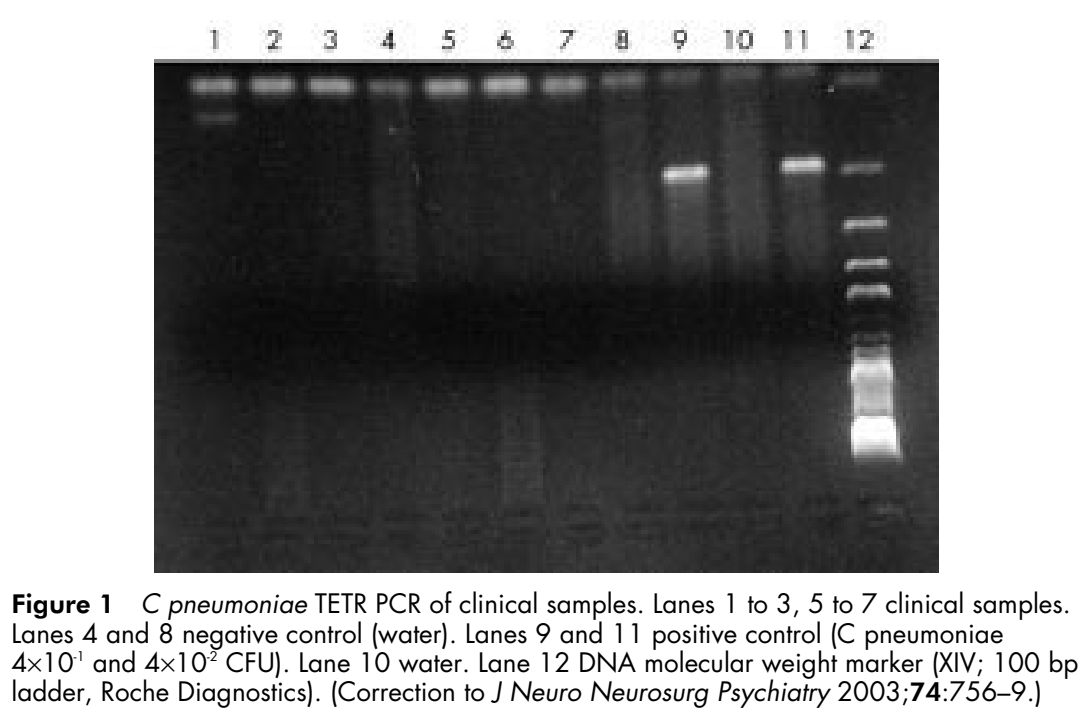

Figure 1 C pneumoniae TETR PCR of clinical samples. Lanes 1 to 3,5 to 7 clinical samples. Lanes 4 and 8 negative control (water). Lanes 9 and 11 positive control (C pneumoniae $4 \times 10^{-1}$ and $\left.4 \times 10^{-2} \mathrm{CFU}\right)$. Lane 10 water. Lane 12 DNA molecular weight marker (XIV; $100 \mathrm{bp}$ ladder, Roche Diagnostics). (Correction to J Neuro Neurosurg Psychiatry 2003;74:756-9.) patients there is nothing we can do for you.

N Bogduk, J Govind, W King

Royal Newcastle Hospital, Australia

Correspondence to: Professor N Bogduk, Department of Clinical Research, Royal Newcastle Hospital, Newcastle, NSW 2300, Australia

\section{Reference}

1 Lord SM, Barnsley L, Wallis BM, et al. Percutaneous radio-frequency neurotomy for chronic cervical zygapophysial joint pain. $N$ Engl J Med 1998;335:1721-6.

\section{CORRECTIONS}

In the neurological picture of the June issue (Komotar JR, Clatterbuck RE. Coccidiomycosis of the brain, mimicking en plaque meningioma. $J$ Neurol Neurosurg Psychiatry 2003;74:806) the initials of the first author were reversed; his name should read as Komotar RJ.

The ordering of the authors in the letter by Soragna D, Tupler R, Ratti et al in the June issue (An Italian family affected by NasuHakola disease with a novel genetic mutation in the TREM2 gene. J Neurol Neurosurg Psychiatry 2003;74:825-6) is incorrect, it should be as follows: D Soragna, L Papi, MT Ratti, R Sestini, R Tupler, L Montalbetti.

The ordering of the authors in the letter by De Tiège, Laureys, Goldman, et al in the July issue (Regional cerebral glucose metabolism in akinetic catatonia and after remission. $J$ Neurol Neurosurg Psychiatry 2003;74:1003-4) is incorrect, it should read as follows: X De Tiège, JC Bier, I Massat, S Laureys, F Lotstra, J Berré, J Mendlewicz, S Goldman.

In the June issue of JNNP fig 1 of the paper by Cagli S, Oktar N, Dalbasti T, et al (Failure to detect Chlamydia pneumoniae DNA in cerebral aneurysmal sac tissue with two different polymerase chain reaction methods. J Neurol Neurosurg Psychiatry 2003;74:756-9) was incorrect. The following figure is the correct image that should have been published. we feel it would be dishonest of us to tell our 